\title{
Awareness of Diagnosis in Patients with Cancer: A Study from Egypt
}

\author{
Ahmad M. Hammad ${ }^{1}$, Dina E. Farag', Mostafa S. El-Haddad ${ }^{2}$, Rasha Haggag', Omar O. Zaki ${ }^{2}$ \\ Noha Y. Ibrahim ${ }^{2}$, Samy A. Alsirafy ${ }^{1}$ \\ ${ }^{1}$ Palliative Medicine Unit, Kasr Al-Ainy Center of Clinical Oncology \& Nuclear Medicine, Kasr Al- \\ Ainy School of Medicine, Cairo University, ${ }^{2}$ Clinical Oncology Department, Kasr Al-Ainy Center of \\ Clinical Oncology \& Nuclear Medicine, Kasr Al-Ainy School of Medicine, Cairo University, ${ }^{3}$ Medical \\ Oncology Department, Faculty of Medicine, Zagazig University, Egypt.
}

Background: One of the barriers to cancer care is the inadequate communication with patients and their families. In Egypt, it is believed that culture is against the honest disclosure of cancer diagnosis. So, the majority of cancer patients are usually unaware of their diagnosis.

Aim: To study cancer diagnosis awareness among a group of Egyptian patients and the factors contributing to their unawareness.

Methods: The study included 119 cancer patients from two cancer centers; one in Cairo and the other in Sharkia. Patients were asked about their illness and who answered as having "cancer/tumor" or mentioned the name of cancer considered aware.

Results: The majority of our patients $(100 / 119,84 \%)$ were aware of their cancer diagnosis. Their answer was "cancer" in $31 \%$, "tumor" in $60 \%$ and the name of cancer in $9 \%$. While, a minority $(19 / 119,16 \%)$, were unaware of their cancer diagnosis. They were more likely to be less educated, older in age, males, unmarried, and from Sharkia. None of the studied variables associated significantly with awareness.

Conclusions: The results of this study suggest that most cancer patients in Egypt are aware of their diagnosis. This may be due to a change in the Egyptian cultural attitude towards truth telling and a shift in the approach of care from paternalistic to patient-centred.

Key words: Cancer Diagnosis, Disclosure, Culture, Attitude, Egypt

Corresponding Author: Dr. Ahmad M. Hammad, Palliative Medicine Unit, Kasr Al-Ainy Center of Clinical Oncology \& Nuclear Medicine (NEMROCK) Kasr Al-Ainy School of Medicine, Cairo University E-mail: hammad_onc@yahoo.com

Submitted: 29-October-2016, Revised: 18-November-2016, Accepted: 20-Novebmer-2016

\section{INTRODUCTION}

Worldwide, cancer care is shifting from a paternalistic approach to a patient-centered one that respects the patient's autonomy. This necessitates meeting the information needs of cancer patients including cancer diagnosis disclosure.

The increasingly recognized importance of disclosing information about cancer diagnosis to patients changed the attitudes towards cancer diagnosis disclosure over the last few decades ${ }^{1-3}$. Six decades ago, only $10 \%$ of physicians in the United States preferred to tell patients their cancer diagnosis ${ }^{1}$. Two decades later, this percentage increased to $97 \%{ }^{2}$. A Lebanese study, during the period from 1995 to 2015, revealed an increase in the percentage of physicians who prefers cancer diagnosis disclosure from $47 \%$ to $69 \%{ }^{3}$.

Factors which influence the attitude and practice of truth-telling in the cancer care setting include culture and physician-related factors ${ }^{4,5}$. Furthermore, medical professionals face the fact that patients are different regarding their desire to know or to ignore their cancer diagnosis ${ }^{6}$.
In many Eastern Mediterranean countries, cancer diagnosis is still accompanied with social stigma and misunderstanding due to believe of incurability. The majority of these countries did not develop guidelines toward truth telling to a patient suffering from cancer ${ }^{7}$.

Data emanating from Egypt are limited. Since, only few studies investigated issues related to cancer diagnosis disclosure in the Egyptian cancer care setting $^{5,8,9}$. Therefore, we aimed to evaluate prevalence of and factors associated with unawareness of diagnosis among Egyptian cancer patients.

\section{METHODS}

During the period from November 2014 to March 2015 , this prospective observational cross-sectional cohort was conducted in two Egyptian cancer centres. The first is in Cairo (Kasr Al-Ainy Center of Clinical Oncology \& Nuclear Medicine, Kasr Al-Ainy School of Medicine, Cairo University) and the other in Sharkia (Medical Oncology Department, Faculty of Medicine, Zagazig University). 
The inclusion criteria of patients were being an adult ( $>18$ years), having a confirmed cancer diagnosis, being able to communicate and to give a verbal informed consent.

In order to determine the awareness of diagnosis, patients were asked about the disease for which they are receiving treatment in the corresponding facility. Patients were considered aware of their diagnosis if they answered using the words "cancer" or "tumor" or by mentioning the name of cancer.

Besides enquiring about the diagnosis, the following data were collected: age, gender, marital status, educational level and primary cancer diagnosis.

Statistical comparison for categorical variables was performed using the Chi-square test. Fisher's exact test was used when expected frequencies were low. A p-value $<0.05$ was considered significant. Statistical methods were performed using the Statistical Package for the Social Sciences for Windows, version 14.0 (SPSS Inc, Chicago, Illinois).

\section{RESULTS}

A total of 119 patients with confirmed cancer diagnosis were interviewed.

The characteristics of patients are shown in Table 1.

Patients' awareness of their cancer diagnosis and how they expressed their awareness are illustrated in Figure 1.

The majority $(100 / 119,84 \%)$ of cancer patients were aware of their diagnosis.

Among the 100 aware patients, 31\% answered that they have "cancer", $60 \%$ answered that they have "tumor" and 9\% named the cancer.

Table 2 shows the relation between the studied variables and awareness of cancer diagnosis. No significant association found between any of the studied variables and the awareness of cancer diagnosis.

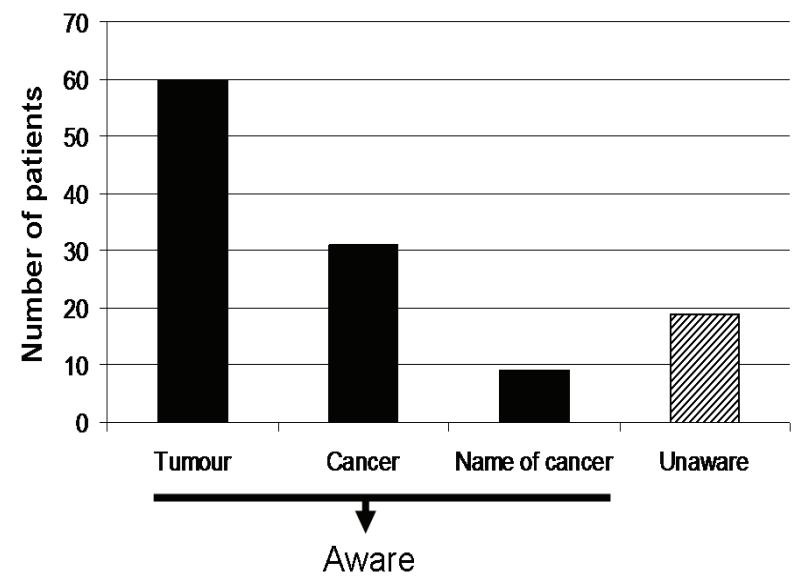

Figure 1: Cancer patients' awareness of their cancer diagnosis.
Table 1: Patients' characteristics.

\begin{tabular}{lll}
\hline & n. & $\%$ \\
\hline Age (years) & & \\
\hline \multicolumn{1}{c}{ Median (range) } & $43(18-95)$ \\
\hline Sex & & \\
\hline Female & 64 & 54 \\
\hline Male & 55 & 46 \\
\hline Marital status & & \\
\hline Married & 98 & 82 \\
\hline Single & 10 & 8 \\
\hline Widow & 8 & 7 \\
\hline Divorced & 3 & 3 \\
\hline Education & & \\
\hline Illiterate & 40 & 34 \\
\hline Can read and write & 11 & 9 \\
\hline Less than high school & 18 & 15 \\
\hline High school & 39 & 33 \\
\hline University & 11 & 9 \\
\hline Governorate & 33 & 72 \\
\hline Cairo & & \\
\hline Sharkia & & \\
\hline Pro & & \\
\hline
\end{tabular}

Primary cancer

\begin{tabular}{lcc} 
Hematological & & \\
\hline Leukemias & 34 & 29 \\
\hline Others & 21 & 18 \\
\hline Solid & 14 & 12 \\
\hline Breast & 14 & 12 \\
\hline Colorectal & 5 & 4 \\
\hline Lung & 5 & 4 \\
\hline Central nervous system & 5 & 4 \\
\hline Sarcomas & 21 & 18
\end{tabular}

\section{DISCUSSION}

Taking into account the ongoing advances in research communication in the cancer care setting, little attention in our region has been paid to this area of research. To the best of our knowledge, this is the first study to investigate the awareness of Egyptian cancer patients of their diagnosis. Also, this is one of few studies addressing communication with cancer patients in Egypt.

Attitudes, perceptions, and beliefs about truth telling regarding a cancer diagnosis or prognosis seem to be very controversial in the Middle East region. 
Table 2: The relation between variables and cancer diagnosis awareness

\begin{tabular}{|c|c|c|c|}
\hline \multirow{2}{*}{ Variable } & \multicolumn{2}{|c|}{$\begin{array}{c}\text { Aware of diagnosis, } \\
\text { n (\%) }\end{array}$} & \multirow{2}{*}{ P-value } \\
\hline & $\begin{array}{c}\text { No } \\
(n=19)\end{array}$ & $\begin{array}{c}\text { Yes } \\
(\mathrm{n}=100)\end{array}$ & \\
\hline \multicolumn{4}{|l|}{ Age } \\
\hline$<35$ & $4(11)$ & $32(89)$ & \multirow[t]{3}{*}{0.4} \\
\hline$\geq 35-<60$ & $10(16)$ & $53(84)$ & \\
\hline$\geq 60$ & $5(25)$ & $15(75)$ & \\
\hline \multicolumn{4}{|l|}{ Sex } \\
\hline Female & $8(13)$ & $56(88)$ & \multirow[t]{2}{*}{0.27} \\
\hline Male & $11(20)$ & $44(80)$ & \\
\hline \multicolumn{4}{|l|}{ Education } \\
\hline No formal education & $10(20)$ & $41(80)$ & \multirow[t]{3}{*}{0.27} \\
\hline $\begin{array}{l}\text { Up to high school } \\
\text { formal education }\end{array}$ & $9(16)$ & $48(84)$ & \\
\hline University & 0 & $11(100)$ & \\
\hline \multicolumn{4}{|l|}{ Marital status } \\
\hline Married & $15(15)$ & $83(85)$ & \multirow[t]{2}{*}{0.67} \\
\hline Others & $4(19)$ & $17(81)$ & \\
\hline \multicolumn{4}{|l|}{ Governorate } \\
\hline Cairo & $12(14)$ & $74(86)$ & \multirow[t]{2}{*}{0.33} \\
\hline Sharkia & $7(21)$ & $26(79)$ & \\
\hline \multicolumn{4}{|l|}{ Primary cancer } \\
\hline Haematological & $8(13)$ & $56(88)$ & \multirow[t]{2}{*}{0.27} \\
\hline Solid & $11(20)$ & $44(80)$ & \\
\hline
\end{tabular}

The social structure of the region is based usually on the importance of the family bonds instead of the individual autonomy ${ }^{7}$.

In our study, the majority of patients (84\%) were aware of their cancer diagnosis, consistent with the results of another study from Egypt that included family caregivers $^{8}$.

The prevalence of awareness of diagnosis among cancer patients varies widely from a country to another. Our results are very close to that of a Norwegian population-based survey in which $80 \%$ of patients with the diagnosis of invasive cancer said that they have or have had cancer ${ }^{10}$. On the other hand, the prevalence was much lower than ours and was only $37 \%$ and $41 \%$ in two Greek studies ${ }^{11,12}$. In Turkey, another Mediterranean country, the prevalence of awareness was $45 \%, 46 \%$ and $88 \%$ in three studies ${ }^{13-15}$. From the aforementioned, we find that the truth disclosure practices are not homogeneous worldwide, with observed improvement during the last two decades. Many factors may have contributed to such variation in the prevalence of awareness among countries. Culture and medico-legal handling of communication issues with cancer patients may have contributed to that variability.
In the current study; older patients, males and those with solid tumor diagnosis were more likely to be unaware of their diagnosis. However, there was no statistically significant relation. This may be explained by the small sample size in our study.

Studies from other countries found that some factors correlated significantly with diagnosis awareness. A Chinese study revealed that age, gender, cancer diagnosis and stage together with the number of health care facilities visited by patients correlated significantly with diagnosis awareness ${ }^{16}$. In Greek studies, hospital specialization in oncology, admission to a medical department, female gender, higher education and younger age were associated significant higher prevalence of diagnosis awareness ${ }^{11,12}$.

In Middle Eastern countries, physicians were generally reluctant to disclose cancer diagnosis to their patients and prefer to involve family members ${ }^{7}$. In a Kuwaiti survey, $67 \%$ of physicians had a tendency to full disclosure of information to cancer patients ${ }^{17}$. On the other hand, in Saudi Arabia, 75\% of physicians preferred discussing the information with the relatives rather than patients ${ }^{18}$.

A minority of our patients were unaware of their cancer diagnosis which may be attributed to physicians' reluctance to disclose cancer diagnosis to their patients. They think that truth-telling may harm the patient and so prefer to inform a family member instead of the patient. In context, two decades ago, El-Ghazali investigated the opinion of 100 Egyptian general surgeons on disclosure of cancer diagnosis "telling the truth" to their patients. She divided the cohort of surgeons into senior surgeons with more than 20 years of experience and junior surgeons with less than 10 years of experience. Only $6 \%$ of senior surgeons preferred to inform the patient first about cancer diagnosis. Moreover, none of the junior surgeons preferred telling the truth ${ }^{5}$. Regarding full information disclosure to the patient, $30 \%$ of senior surgeons preferred full disclosure versus $4 \%$ of junior surgeons $(p<0.001)^{5}$.

Noteworthy, some of the health care professionals believe that disclosing diagnosis to cancer patients may have a negative impact on their quality of life and may associate with psychological morbidity, which is not true $^{19-21}$. As a study from Turkey included 100 cancer patients of whom 44 were unaware of diagnosis and the investigators measured the quality of their lives using the European Organization for Research and Treatment of Cancer Quality of Life Questionnaire (EORTC QLQ-C30). The QLQ-C30 scales' scores did not differ according to the awareness of cancer diagnosis ${ }^{19}$. Among Taiwanese cancer patients, truth-telling was associated with significantly less uncertainty and anxiety with no significant impact on their spiritual wellbeing ${ }^{20}$.

Another factor that may have contributed to the unawareness of some of our patients is their families' negative attitude towards truth-telling. In context, a 
recent Egyptian research revealed that some of family caregivers preferred not to disclose cancer diagnosis to patients, but, the majority preferred truth-telling ${ }^{8}$.

Besides our cultural and religious codes, legislations should assure our patient's right to have an informed consent which should be applied in the Middle Eastern countries.

Finally, communication with cancer patients and their families in cancer care setting needs much research attention in our region.

\section{CONCLUSIONS}

The majority of Egyptian patients with cancer are aware of their diagnosis. This may be due a change in the cultural attitude towards truth telling in Egypt. In addition, this may indicate a transition in the approach of cancer care in Egypt from paternalistic to patient-centred. Communication with cancer patients and their families in our region is an under-researched area.

\section{ACKNOWLEDGMENT}

Results were presented as a poster at the European Cancer Congress 2015(\#p308).

\section{REFERENCES}

1. Oken D. What to tell cancer patients: a study of medical attitudes. JAMA. 1961; 175:1120-1128.

2. Novack DH, Plumer R, Smith RL, Ochitill H, Morrow GR, Bennett JM. Changes in physicians' attitudes toward telling the cancer patient. JAMA. 1979; 241(9): 897-900.

3. Naji F, Hamadeh G, Hlais S, Adib S. Truth Disclosure to Cancer Patients: Shifting Attitudes and Practices of Lebanese hysicians. AJOB Empir Bioeth. 2015; 6(3): 41-49.

4. Mystakidou K, Parpa E, Tsilila E, Katsouda E, Vlahos L. Cancer information disclosure in different cultural contexts. Support Care Cancer. 2004; 12(3): 147-154.

5. El-Ghazali, S. Is it wise to tell the truth, the whole truth, and nothing but the truth to a cancer patient? Ann N Y Acad Sci. 1997; 809: 97-108.

6. van der Molen B. Relating information-needs to the cancer experience. 1. Jenny's story: A cancer narrative.Eur J Cancer Care (Engl). 2000; 9: 41-47.

7. Bou Khalil R. Attitudes, beliefs and perceptions regarding truth disclosure of cancer-related information in the Middle East: a review. Palliat Support Care. 2013; 11(1): 69-78.
8. Alsirafy SA, Abdel-Kareem SS, Ibrahim NY, Abolkasem MA, Farag DE. Cancer diagnosis disclosure preferences of family caregivers of cancer patients in Egypt. Psychooncology. 2016 Jun 30. doi: 10.1002/pon.4206. [Epub ahead of print].

9. Ali NS, Khalil HZ, Yousef W. A comparison of American and Egyptian cancer patients' attitudes and unmet needs. Cancer Nurs. 1993; 16(3): 193-203.

10. Nord C, Mykletun A, Fosså SD. Cancer patients' awareness about their diagnosis: a population-based study. J Public Health Med. 2003; 25(4): 313-317.

11. Iconomou G1, Viha A, Koutras A, Vagenakis AG, Kalofonos HP. Information needs and awareness of diagnosis in patients with cancer receiving chemotherapy: a report from Greece. Palliat Med. 2002; 16: 315- 321.

12. Brokalaki EI, Sotiropoulos GC, Tsaras K, Brokalaki H. Awareness of diagnosis and information-seeking behavior of hospitalized cancer patients in Greece. Support Care Cancer. 2005; 13: 938-942.

13. Atesci FC, Baltalarli B, Oguzhanoglu NK, Karadag F, Ozdel O, Karagoz N. Psychiatric morbidity among cancer patients and awareness of illness. Support Care Cancer. 2004; 12(3): 161-167.

14. Ateşci FC, Oğuzhanoğlu NK, Baltalarli B, Karadağ F, Ozdel O, Karagöz N. Psychiatric disorders in cancer patients and associated factors. Turk Psikiyatri Derg. 2003; 14(2): 145-152.

15. Sen M. Communication with cancer patients. The influence of age, gender, education, and health insurance status. Ann N Y Acad Sci. 1997; 809:514-524.

16. Wang DC1, Guo CB, Peng X, Su YJ, Chen F. Is therapeutic non-disclosure still possible? A study on the awareness of cancer diagnosis in China. Support Care Cancer. 2011; 19(8): 1191-1195.

17. Qasem AA, Ashour TH, Al-Abdulrazzaq HK, Ismail ZA. Disclosure of cancer diagnosis and prognosis by physicians in Kuwait. Int J Clin Pract. 2002; 56(3): 215-218.

18. Mobeireek AF, al-Kassimi FA, al-Majid SA, AlShimemry A. Communication with the seriously ill: physicians' attitudes in Saudi Arabia. J Med Ethics. 1996; 22(5): 282-285.

19. Bozcuk H, Erdoğan V, Eken C, et al. Does awareness of diagnosis make any difference to quality of life? Determinants of emotional functioning in a group of cancer patients in Turkey. Support Care Cancer. 2002; 10(1): 51-57.

20. Kao CY, Cheng SY, Chiu TY, Chen CY, Hu WY. Does the awareness of terminal illness influence cancer patients' psycho-spiritual state, and their DNR signing: a survey in Taiwan. Jpn J Clin Oncol. 2013; 43(9): 910-916.

21. Wang DC, Guo CB, Peng X, Su YJ. Psychological morbidity and health-related quality of life in patients with differing awareness of cancer diagnosis: a cross-sectional study. Psychooncology. 2014; 23(9): 975-980. 